\title{
Existence and Uniqueness of Weak Solution for Weighted p-bilaplacian (p-biharmonic)
}

\author{
Bassam Al-Hamzah, Naji Yebari \\ University Abdelmalek Essaadi, Faculty of Sciences, Department of Mathematics, Tetouan, Morocco
}

Email address:

bassamalhamzah@yahoo.com (B. Al-Hamzah),nyebari@fst.ac.ma (N. Yebari), yebarinaji@gmail.com (N. Yebari)

\section{To cite this article:}

Bassam Al-Hamzah, Naji Yebari. Existence and Uniqueness of Weak Solution for Weighted p-bilaplacian (p-biharmonic). American Journal of Applied Mathematics. Vol. 3, No. 6, 2015, pp. 283-287. doi: 10.11648/j.ajam.20150306.17

Abstract: This paper deals with the equation $-\Delta_{p}^{2} u+\lambda \ell(x)|u|^{p-2} u=f(x, y)$ in bounded domain $\Omega \in \mathfrak{R}^{N}$. Relying on Browder theorem, under conditions of the monotonous function $\mathrm{f}$. We obtained the existence and uniqueness of weak solutions for the weighted p-bilaplacian boundary value of the form: $(P)\left\{\begin{array}{l}-\Delta_{p}^{2} u+\lambda \ell(x)|u|^{p-2} u=f(x, u) \text { in } \Omega \\ u \in W_{0}^{2, p}(\Omega)\end{array}\right.$

Keywords: Weak Solutions, p-biharmonic Operator, Browder Theorem

\section{Introduction}

In this paper, we are concerned with the existence and uniqueness of weak solution for a weighted p-bilaplacian boundary value of the form:

$$
(P)\left\{\begin{array}{l}
-\Delta_{p}^{2} u+\lambda \ell(x)|u|^{p-2} u=f(x, u) \text { in } \Omega \\
u \in W_{0}^{2, p}(\Omega)
\end{array}\right.
$$

Let $\Omega$ be a bounded domain in $\mathfrak{R}^{N}$ and $f: \Omega \times \mathfrak{R} \rightarrow \mathfrak{R}$ be a Caratheodry (CAR) Satisfies the following:

(H1) $f\left(x, s_{1}\right) \leq f\left(x, s_{2}\right)$ for a.e. $x \in \Omega$ and $s_{1}, s_{2} \in \mathfrak{R}$, $s_{1} \geq s_{2}$.

$$
|f(x, s)| \leq f_{0}(x)+c|s|^{p-1} \text { that there exists }
$$

$f_{0} \in L^{p^{\prime}}(\Omega), c>0$.

Here $\quad \Delta_{p}^{2} u=\Delta\left(|\Delta u|^{p-2} \Delta u\right)$, and $\quad N>1,1<p<\infty, \lambda \in \mathfrak{R}$ $\ell \in L^{r}(\Omega), \ell \neq 0$, and $r=r(N, p)$

Satisfying the conditions:

$$
\left(\begin{array}{l}
H_{3}
\end{array}\right)\left\{\begin{array}{l}
r>\frac{N}{2 p} \text { if } \frac{N}{p}>2 \\
r>p \text { if } \frac{N}{p}=2 \\
r=1 \text { if } \frac{N}{p}<2
\end{array}\right.
$$

We assume that $\left|\Omega_{\ell}^{+}\right| \neq 0$, where $\Omega_{\ell}^{+}=\{x \in \Omega / \ell(x)>0\}$.

Our paper is organized as follow: Section 2 contains some basic definitions concerning the nonlinear operators that will be used throughout the paper. Also, we introduce the space setting of the problem and give some basic characteristics, as the equivalent norm and embedding results. In section 3 we state the main result on the existence and uniqueness of weak solutions of the problem $(\mathrm{P})$.

The existence of solutions for the nonlinear eigenvalue problem with p-biharmonic see [3].

\section{Preliminaries and Space Setting}

First, we introduce some basic definitions concerning the nonlinear operators which we use extensively in this paper 3 .

Definition 2.1 [4] Let $\mathrm{A}: \mathrm{V} \rightarrow \mathrm{V}$ ' be an operator on a real Banach space V. We say that the operator $A$ is :

(i) bounded iff it maps bounded sets into bounded i.e. for each $r>0$ there exists $M>0$ ( $M$ depending on $r$ ) such that

$$
\|u\| \leq r \Rightarrow\|A(u)\| \leq M, \forall u \in V
$$

(ii) Coercive : iff 


$$
\lim _{\|u\| \rightarrow \infty} \frac{\langle A(u), u\rangle}{\|u\|}=\infty
$$

(iii) Monotone iff $<\mathrm{A}\left(\mathrm{u}_{1}\right)-\mathrm{A}\left(\mathrm{u}_{2}\right), \mathrm{u}_{1}-\mathrm{u}_{2}>\geq 0, \forall \mathrm{u}_{1}, \mathrm{u}_{2} \in$ $\mathrm{V}$.

(iv) Strictly monotone iff

$<\mathrm{A}\left(\mathrm{u}_{1}\right)-\mathrm{A}\left(\mathrm{u}_{2}\right), \mathrm{u} 1-\mathrm{u}_{2}>>0$, for all $\mathrm{u} 1, \mathrm{u}_{2} \in \mathrm{V}, \mathrm{u}_{1} \neq \mathrm{u}_{2}$.

(v) Strongly monotone iff there exists $\mathrm{k}>0$,

$<\mathrm{A}\left(\mathrm{u}_{1}\right)-\mathrm{A}\left(\mathrm{u}_{2}\right), \mathrm{u}_{1}-\mathrm{u}_{2}>\geq k\left\|u_{1}-u_{2}\right\|$, for all $\mathrm{u}_{1}, \mathrm{u}_{2} \in \mathrm{V}$, $\mathrm{u}_{1} \neq \mathrm{u}$;

(vi) Continuous iff un $\rightarrow \mathrm{u}$ implies $\mathrm{A}($ un $) \rightarrow \mathrm{A}(\mathrm{u})$, for all un , $\mathrm{u} \in \mathrm{V}$.

(vii) Strongly continuous iff un $\stackrel{w}{\longrightarrow} \mathrm{u}$ implies $\mathrm{A}$ (un $) \rightarrow \mathrm{A}(\mathrm{u})$, for all $\mathrm{un}, \mathrm{u} \in \mathrm{V}$.

(viii) Demi continuous iff un $\rightarrow \mathrm{u}$ implies $\mathrm{A}$ (un ) $\stackrel{w}{\longrightarrow}$ $\mathrm{A}(\mathrm{u})$, for all $\mathrm{un}, \mathrm{u} \in \mathrm{V}$.

Theorem 2.1(Browder) [4]

Let $\mathrm{A}$ be a reflexive real Banach space. Moreover let A : V $\rightarrow \mathrm{V}$ ' be an operator which is: bounded, demicontinuous, coercive, and monotone on the space $\mathrm{V}$. Then, the equation $\mathrm{A}(\mathrm{u})=\mathrm{f}$ has at least one solution $\mathrm{u} \in \mathrm{V}$ for each $\mathrm{f} \in \mathrm{V}$ '. If moreover, $\mathrm{A}$ is strictly monotone operator, then the equation (P) has precisely one solution $\mathrm{u} \in \mathrm{V}$ for every $\mathrm{f} \in \mathrm{V}$ '.

Proposition 2.1 [6] For any bounded domain $\Omega$ and $1<$ $\mathrm{p}<\infty, \Delta_{p}^{2}$ satisfies the following:

(i) $\Delta_{p}^{2}$ is an hemicontinuous operator from $W_{0}^{2, p}(\Omega)$ into $W^{-2, p^{\prime}}(\Omega)$.

(ii) $\Delta_{p}^{2}$ is a bounded monotonous, and coercive operator.

(iii) $\Delta_{p}^{2}: W_{0}^{2, p}(\Omega) \rightarrow W^{-2, p^{\prime}}(\Omega)$ is a bicontinuous operator.

Proof

See [6].

The norm $\|\Delta$.$\| is uniformly equivalent on W_{0}^{2, p}$ to the usual norm of $W_{0}^{2, p}$.

\section{Existence and Uniqueness Results}

In this section, using Browder theorem, we prove the existence and uniqueness of weak solution for equation (P).

Definition 3.1 We say that $u \in W_{0}^{2, p}(\Omega)$ is a weak solution to equation $(\mathrm{P})$ if

$$
\int_{\Omega}|\Delta u|^{p-2} \Delta u \Delta \varphi+\lambda \int_{\Omega} \ell(x)|u|^{p-2} u \varphi=\int_{\Omega} f(x, u) \varphi, \text { forall } \varphi \in C_{0}^{\infty}(\Omega)
$$

Our main results concerning problem $(\mathrm{P})$ is the following theorem :

Theorem 3.1 Let $\mathrm{p} \geq 2, \lambda>0$ and $\mathrm{f}(\mathrm{x}, \mathrm{u}) \in \mathrm{CAR}(\Omega \times \mathrm{R})$ satisfy $\left(\mathrm{H}_{1}\right),\left(\mathrm{H}_{2}\right)$ and $\left(\mathrm{H}_{3}\right)$. Then problem $(\mathrm{P})$ has a unique weak solution.

Proof:

We define for $\lambda>0$ the operator A: $W_{0}^{2, p}(\Omega) \rightarrow W^{-2, p^{\prime}}(\Omega)$, as $\mathrm{A}=\mathbf{J}+\lambda \mathrm{G}-\mathrm{F}$, where the

Operators $\mathrm{J}: W_{0}^{2, p}(\Omega) \rightarrow W^{-2, p^{\prime}}(\Omega), \mathrm{G}: W_{0}^{2, p}(\Omega) \rightarrow W^{-2, p^{\prime}}(\Omega)$ and $\mathrm{F}: \Omega \times W_{0}^{2, p}(\Omega) \rightarrow W^{-2, p^{\prime}}(\Omega)$ are given by

$$
\begin{aligned}
& \langle J(u), \varphi\rangle=\int_{\Omega}|\Delta u|^{p-2} \Delta u \Delta \varphi \\
& \langle G(u), \varphi\rangle=\int_{\Omega} \ell(x)|u|^{p-2} u \varphi
\end{aligned}
$$

And

$$
\langle F(u), \varphi\rangle=\int_{\Omega} f(x, u) \varphi
$$

for all $\mathrm{u}, \varphi \in W_{0}^{2, p}(\Omega)$. Thus, to find a weak solution of (P) is equivalent to finding $u \in W_{0}^{2, p}(\Omega)$ which satisfies the operator equation $\mathrm{A}(\mathrm{u})=0$. Now, we have the following properties of the operators $\mathrm{J}, \mathrm{G}$, and $\mathrm{F}$ :

a) J, G and F are well defined. Using Holder's inequality, we have

$$
\langle J(u), \varphi\rangle=\int_{\Omega}|\Delta u|^{p-2} \Delta u \Delta \varphi
$$

$$
\begin{gathered}
|\langle J(u), \varphi\rangle| \leq \int_{\Omega}|\Delta u|^{p-1}|\Delta \varphi| d x \leq\left(\int_{\Omega}|\Delta u|^{p}\right)^{\frac{1}{p^{\prime}}}\left(\int_{\Omega}|\Delta \varphi|^{p}\right)^{\frac{1}{p}}<\infty . \\
\langle G(u), \varphi\rangle=\int_{\Omega} \ell(x)|u|^{p-2} u \varphi
\end{gathered}
$$

First case: $\frac{N}{p}>2$ and $r>\frac{N}{2 p}$. Let $\mathrm{u}, \varphi \in W_{0}^{2, p}(\Omega)$ Вy Hőlder's inequality, we have

$$
\left|\left\langle G(u)^{\rho}, \varphi\right\rangle\right| \leq\|\ell\|_{r}\|u\|_{s}^{p-1}\|\varphi\|_{p_{2}}
$$

Where $\frac{1}{p 2}=\frac{1}{p}-\frac{2}{N}$. and $\mathrm{s}$ is given by:

$$
\frac{p-1}{s}+\frac{1}{p 2}+\frac{1}{r}=1
$$

Therefore

$$
\frac{p-1}{s}=1-\frac{1}{r}-\frac{1}{p 2}>1-\frac{2 p}{N}-\frac{1}{p 2}=\frac{p-1}{p 2} .
$$

The nit suffices to take $\max (1, p-1)<s<p_{2}$ so that $\mathrm{G}$ is well defined.

Second case: $\frac{N}{p}=2$, and $r>p$. In this case $W_{0}^{2, p}(\Omega)$ embedded into the space $L^{q}(\Omega)$, for any $q \in[p,+\infty[$, there 
is $q \geq p$ such that:

$$
\frac{1}{q}+\frac{1}{r}+\frac{p-1}{p}=\frac{1}{q}+\frac{1}{r}+\frac{1}{p^{\prime}}=1
$$

We obtain that $\frac{1}{q}=\frac{1}{p}-\frac{1}{r} \leq \frac{1}{p}$. By Hölder's inequality, we arrive at:

$$
|\langle G(u), \varphi\rangle| \leq\|\ell\|_{r}\|u\|_{p}^{p-1}\|\varphi\|_{q}
$$

For any $u, \varphi \in W_{0}^{2, p}(\Omega)$, and $\mathrm{G}$ is well defined.

Third case: $\frac{N}{p}<2$, and $r=1$. In this case $W_{0}^{2, p}(\Omega)$ embedded into the space $C(\bar{\Omega}) \cap L^{\infty}(\Omega)$ Then for any $u, \varphi \in W_{0}^{2, p}(\Omega)$ we have:

$$
|\langle G(u), \varphi\rangle| \leq\|\ell\|_{1}\|u\|_{\infty}^{p-1}\|\varphi\|_{\infty}<\infty
$$

And $\mathrm{G}$ is well defined.

And

$$
\begin{gathered}
\langle F(u), \varphi\rangle=\int_{\Omega} f(x, u) \varphi d x \\
|\langle F(u), \varphi\rangle| \leq \int_{\Omega}|f(x, u) \varphi| \leq \int_{\Omega}\left(f_{0}(x)+c|u|^{p-1}\right)|\varphi| .
\end{gathered}
$$

By Hőlder's inequality,

$|\langle F(u), \varphi\rangle| \leq\left(\int_{\Omega}\left|f_{0}(x)\right|^{p^{\prime}}\right)^{\frac{1}{p^{\prime}}}\left(\int_{\Omega}|\varphi|^{p}\right)^{\frac{1}{p}}+c\left(\int|u|^{p}\right)^{\frac{1}{p^{\prime}}}\left(\int_{\Omega}|\varphi|^{p}\right)^{\frac{1}{p}}<\infty$,

And hence J, G, F are well defined.

b) $\mathrm{G}$ is completely continuous. Let $\left(u_{n}\right) \subset W_{0}^{2, p}(\Omega)$ be as equence such that

un $\rightarrow \mathrm{u}$ weakly in $W_{0}^{2, p}(\Omega)$. We have to show that $\mathrm{G}$ (un) $\rightarrow \mathrm{G}(\mathrm{u})$ strongly in $W^{-2, p^{\prime}}(\Omega)$, i .e.

$$
\sup _{\substack{\varphi \in W_{0}^{2, p} \\\|\Delta \varphi\|_{p} \leq 1}}\left|\int_{\Omega} \ell\left(\left|u_{n}\right|^{p-2} u_{n}-|u|^{p-2} u\right) \varphi d x\right| \underset{\rightarrow 0, a s, n \rightarrow+\infty}{\rightarrow}
$$

For $\frac{N}{p}>2, r>\frac{N}{2 p}$. Let $\mathrm{s}$ be as $\max (1, p-1)<s<p_{2}$ and $\frac{1}{p_{2}}=\frac{1}{p}-\frac{2}{N}$ and $\frac{p-1}{s}+\frac{1}{p_{2}}+\frac{1}{r}=1$.

$$
\begin{aligned}
& \sup _{\substack{\varphi \in W_{0}^{2, p}(\Omega) \\
\|\Delta \varphi\|_{p} \leq 1}}\left|\int_{\Omega} \ell\left(\left|u_{n}\right|^{p-2} u_{n}-|u|^{p-2} u\right) \varphi d x\right| \\
& \leq \sup _{\substack{\varphi \in W_{0}^{2, p}(\Omega) \\
\|\Delta \varphi\|_{p} \leq 1}}\|\ell\|_{r}\left\|\left|u_{n}\right|^{p-2} u_{n}-|u|^{p-2} u\right\|_{\frac{s}{p-1}}\|\varphi\|_{p_{2}} \\
& \leq C\|\ell\|_{r}\left\|\left|u_{n}\right|^{p-2} u_{n}-|u|^{p-2} u\right\|_{\frac{s}{p-1}}
\end{aligned}
$$

where $\mathrm{C}$ is the constant of Sobolev. On the other hand, the Nemytskii's operator

$$
u \mapsto|u|^{p-2} u \text { is continuous from Ls }(\Omega) \text { into } \frac{s}{L^{p-1}}(\Omega) \quad,
$$
and un $\rightarrow \mathrm{u}$ weakly in $W_{0}^{2, p}(\Omega)$. So, we deduce that un $\rightarrow$ $\mathrm{u}$ strongly in Ls $(\Omega)$ because $\mathrm{s}<\mathrm{p} 2$. Hence $\left\|\left|u_{n}\right|^{p-2} u_{n}-|u|^{p-2} u\right\|_{\frac{s}{p-1}} \rightarrow 0$. as $n \rightarrow+\infty$.

This completes the proof of the claim.

If $\frac{N}{p}=2, r>p$ then :

$$
\begin{aligned}
& \left|\int_{\Omega} \ell\left(\left|u_{n}\right|^{p-2} u_{n}-|u|^{p-2} u\right) \varphi d x\right| \\
\leq & \left.\|\ell\|_{r}|| u_{n}\right|^{p-2} u_{n}-|u|^{p-2} u\left\|_{p}^{p-1}\right\| \varphi \|_{q}
\end{aligned}
$$

Where $\mathrm{q}$ is given $\frac{1}{q}=\frac{1}{p}-\frac{1}{r}$. By Sobolev's embedding there exist $c>0$ such that $\|\varphi\|_{q} \leq c\|\Delta \varphi\|_{p}, \forall \varphi \in W_{0}^{2, p}(\Omega)$. Thus

$$
\begin{aligned}
& \sup _{\substack{\varphi \in W_{0}^{2, p}(\Omega) \\
\|\Delta \varphi\|_{p} \leq 1}}\left|\int_{\Omega} \ell\left(\left|u_{n}\right|^{p-2} u_{n}-|u|^{p-2} u\right) \varphi d x\right| \\
& \leq C\|\ell\|_{r}\left\|\left|u_{n}\right|^{p-2} u_{n}-|u|^{p-2} u\right\|_{p}^{p-1}
\end{aligned}
$$

From the continuity of $u \mapsto|u|^{p-1} u$ from $L^{p}(\Omega)$ into $L^{p^{\prime}}(\Omega)$ and from the compact embedding of $W_{0}^{2, p}(\Omega)$ in $L^{p}(\Omega)$ we have the desired result.

If $\frac{N}{p}<2, r=1, W_{0}^{2, p}(\Omega) \subset C(\bar{\Omega})$, then we obtain :

$$
\sup _{\substack{\varphi \in W_{0}^{2, p}(\Omega) \\\|\Delta \varphi\|_{p} \leq 1}}\left|\int_{\Omega} \ell\left(\left|u_{n}\right|^{p-2} u_{n}-|u|^{p-2} u\right) \varphi d x\right|
$$




$$
\leq C\|\ell\|_{1} \sup _{\bar{\Omega}}\left\|\left|u_{n}\right|^{p-2} u_{n}-|u|^{p-2} u\right\|
$$

Where $\mathrm{C}$ is the constant given by embedding $W_{0}^{2, p}(\Omega)$ of in $\mathrm{C}(\Omega) \cap \mathrm{L} \infty(\Omega)$. It is clear that,

$$
\sup _{\bar{\Omega}}\left\|\left|u_{n}\right|^{p-2} u_{n}-|u|^{p-2} u\right\| \rightarrow 0, \text { as } \mathrm{n} \rightarrow+\infty .
$$

Hence $\mathrm{G}$ is completely continuous, also in this case. $\mathrm{J}$ and $\mathrm{F}$ are bounded operators. Indeed, for every u such that $\|u\|_{W_{0}^{2, p}(\Omega)} \leq M$.

We have

$$
\|J(u)\|_{W^{-2, p^{\prime}}(\Omega)}=\sup _{\mid \varphi \|_{W_{0}, p}, p_{(\Omega)} \leq 1}|\langle J(u), \varphi\rangle| \leq \sup _{\|\varphi\|_{\sigma_{0}^{2}, p(\Omega)} \leq 1 \Omega}|\Delta u|^{p-1}|\Delta \varphi| d x .
$$

Using Hölder's inequality, we obtain

$$
\|J(u)\|_{W^{-2, p^{\prime}}(\Omega)}=\sup _{\|\varphi\|_{\sigma_{0}^{2}, p(\Omega)} \leq 1}\left(\int_{\Omega}|\Delta u|^{p}\right)^{\frac{1}{p^{\prime}}}\left(\int_{\Omega} \mid \Delta \varphi^{p}\right)^{\frac{1}{p}} \leq M^{\frac{p}{p^{\prime}}} .
$$

Also, we get

$$
\begin{gathered}
\|F(u)\|_{W^{-2, p^{\prime}}(\Omega)}=\sup _{\|\varphi\|_{W_{0}^{2}, p(\Omega)}}|\langle F(u), \varphi\rangle| \\
\leq \sup _{\|\varphi\|_{w_{0}^{\prime}, p}(\Omega) \leq 1}\left[\left(\int_{\Omega}\left|f_{0}(x)\right|^{p^{\prime}}\right)^{\frac{1}{p^{\prime}}}+\left(\int_{\Omega}|u|^{(p-1) p^{\prime}}\right)^{\frac{1}{p^{\prime}}}\right]\left(\int_{\Omega}|\varphi|^{p}\right)^{\frac{1}{p}} \\
\leq k\left(\left\|f_{0}\right\|_{L^{p^{\prime}}(\Omega)}+k\|u\|_{W_{0}^{\frac{p}{p^{\prime}}}}^{\frac{p}{p^{2}, p}(\Omega)}\right) \leq k\left(\left\|f_{0}\right\|_{L^{p^{\prime}}(\Omega)}+k M^{\frac{p}{p^{\prime}}}\right)
\end{gathered}
$$

Where $\mathrm{k}$ is the constant of the embedding of $W_{0}^{2, p}(\Omega)$ into $L^{p}(\Omega)$.

c) $\mathrm{J}$ and $\mathrm{F}$ are continuous operators. If $u_{n} \rightarrow u$, in $W_{0}^{2, p}(\Omega)$. Then, we have

$$
\left\|u_{n}-u\right\|_{W_{0}^{2, p}(\Omega)} \rightarrow 0,\left\|\Delta u_{n}-\Delta u\right\|_{L^{p}(\Omega)} \rightarrow 0
$$

Applying Dominated Convergence Theorem, we obtain

$$
\left\|\left|\Delta u_{n}\right|^{p-2} \Delta u_{n}-|\Delta u|^{p-2} \Delta u\right\|_{L^{p}(\Omega)} \rightarrow 0 .
$$

Hence

$$
\begin{aligned}
& \left\|J\left(u_{n}\right)-J(u)\right\|_{W^{-2, p^{\prime}}(\Omega)}=\sup _{\|\varphi\|_{w_{0}^{2}, p(\Omega)} \leq 1}\left|\left\langle J\left(u_{n}\right)-J(u), \varphi\right\rangle\right| \\
& \leq \sup _{|\varphi|_{W_{0}^{2}, p^{\prime}(\Omega)} \leq 1}\left(\int_{\Omega}\left(\left|\Delta u_{n}\right|^{p-2} \Delta u_{n}-|\Delta u|^{p-2} \Delta u\right)^{p^{\prime}}\right)^{\frac{1}{p^{\prime}}}\left(\int_{\Omega}|\varphi|^{p^{2}}\right)^{\frac{1}{p}}
\end{aligned}
$$

$$
\leq k\left(\int_{\Omega}\left(\left|\Delta u_{n}\right|^{p-2} \Delta u_{n}-|\Delta u|^{p-2} \Delta u\right)^{p^{\prime}}\right)^{\frac{1}{p^{\prime}}} \rightarrow 0 \text { as } n \rightarrow \infty .
$$

0 Similarly, we have

$$
\begin{gathered}
\left\|F\left(u_{n}\right)-F(u)\right\|_{W^{-2, p^{\prime}}(\Omega)}=\sup _{\|\varphi\|_{w_{0}^{2}, p^{(}(\Omega)} \leq 1}\left|\left\langle F\left(u_{n}\right)-F(u), \varphi\right\rangle\right| \\
\leq k\left(\int_{\Omega}\left(\left|f\left(x, u_{n}\right)-f(x, u)\right|\right)^{p^{\prime}}\right)^{\frac{1}{p^{\prime}}} \rightarrow 0 \text { as } n \rightarrow \infty .
\end{gathered}
$$

d) Let $p \geq 2, \forall x_{1}, x_{2} \in \Re^{N}$, we have the following inequality (see [8] )

$$
\left|x_{2}\right|^{p} \geq\left|x_{1}\right|^{p}+p\left|x_{1}\right|^{p-2} x_{1}\left(x_{2}-x_{1}\right)+\frac{\left|x_{2}-x_{1}\right|^{P}}{2^{p-1}-1}
$$

Now,

$$
\begin{aligned}
& \langle J(u)-J(\varphi), u-\varphi\rangle=\int_{\Omega}\left[|\Delta u|^{p-2} \Delta u-|\Delta \varphi|^{p-2} \Delta \varphi\right](\Delta u-\Delta \varphi) \\
& =\int_{\Omega}|\Delta u|^{p-2} \Delta u(\Delta u-\Delta \varphi)-\int_{\Omega}|\Delta \varphi|^{p-2} \Delta \varphi(\Delta u-\Delta \varphi)=I_{1}+I_{2}
\end{aligned}
$$

Using (1), we get

$$
I_{1}+I_{2} \geq \frac{2}{p\left(2^{p-1}-1\right)} \int_{\Omega}|\Delta u-\Delta \varphi|^{p}=c(p)\|u-\varphi\|_{W_{0}^{2, p}(\Omega)}^{p},
$$

for $p \geq 2$.

So

$$
\langle J(u)-J(\varphi), u-\varphi\rangle \geq c(p)\|u-\varphi\|_{W_{0}^{2, p}(\Omega)}^{p} \text {, for } p \geq 2 \text {. }
$$

Similarly, we have

$$
\begin{gathered}
\langle G(u)-G(\varphi), u-\varphi\rangle=\int_{\Omega} \ell(x)\left[|u|^{p-2} u-|\varphi|^{p-2} \varphi\right](u-\varphi) \\
\geq \frac{2}{p\left(2^{p-1}-1\right)} \int_{\Omega} \ell(x)|u-\varphi|^{p} \geq c(p)\|u-\varphi\|_{W_{0}^{2, p}(\Omega)}^{p} \geq 0 .
\end{gathered}
$$

Hence,

$$
\langle G(u)-G(\varphi), u-\varphi\rangle \geq 0
$$

Also, we get

$$
\langle F(u)-F(\varphi), u-\varphi\rangle=\int_{\Omega}[f(x, u)-f(x, \varphi)](u-\varphi) .
$$

Since $f$ is decreasing with respect to the second variable, we have

$$
[f(x, u)-f(x, \varphi)](u-\varphi) \leq 0 .
$$


Consequently

$$
\langle F(u)-F(\varphi), u-\varphi\rangle=\int_{\Omega}[f(x, u)-f(x, \varphi)](u-\varphi) \leq 0 .
$$

Equations (2),(3), and (4) imply that

$$
\langle A(u)-A(\varphi), u-\varphi\rangle \geq c(p)\|u-\varphi\|_{W_{0}^{2, p}(\Omega)}^{p}, \text { for } p \geq 2 \text {. (5) }
$$

So A is strongly monotone.

Now, to apply Browder theorem, it remains to prove that A is a coercive operator. From (5), we have

$$
\langle A(u), u\rangle \geq\langle A(0), u\rangle+c(p)\|u\|_{W_{0}^{2, p}(\Omega)}^{p} .
$$

On the other hand

$$
\begin{aligned}
& \langle A(0), u\rangle=\langle J(0), u\rangle+\lambda\langle G(0), u\rangle-\langle F(0), u\rangle=-\int_{\Omega} f(x, 0) u \\
& \geq-\left(\int_{\Omega}\left[f_{0}(x)\right]^{p^{\prime}}\right)^{\frac{1}{p^{\prime}}}\left(\int_{\Omega}|u|^{p}\right)^{\frac{1}{p}} \geq-k\left\|f_{0}\right\|_{L^{p^{\prime}(\Omega)}}\|u\|_{W_{0}^{2, p}(\Omega)},
\end{aligned}
$$

Then

$$
\langle A(u), u\rangle \leq c(p)\|u\|_{W_{0}^{2, p}(\Omega)}^{p}-k\left\|f_{0}\right\|_{L^{p^{\prime}}(\Omega)}\|u\|_{W_{0}^{2, p}(\Omega)} \cdot
$$

So

$$
\lim _{\|u\|_{W_{0}^{2, p}(\Omega)} \rightarrow \infty} \frac{\langle A(u), u\rangle}{\|u\|_{W_{0}^{2, p}(\Omega)}}=\infty .
$$

This proves the coercivity condition and so, the existence of weak solution for (P). The uniqueness of weak solution of (P) is a direct consequence of (5). Suppose that $u, \varphi$ be a weak solutions of (P) such that $u \neq \varphi$. Now, from (5), we have

$$
0=\langle A(u)-A(\varphi), u-\varphi\rangle \geq c(p)\|u-\varphi\|_{W_{0}^{2, p}(\Omega)}^{p} \geq 0 .
$$

Therefore $\mathrm{u}=\varphi$. This completes the proof.

\section{References}

[1] G. A. Afrouzi, S. Mahdavi, Z. Naghizadeh, Existence and Uniqueness of Solution for P Laplacian Dirichlet Problem, ISSN 1749-3889 (print), 1749-3897 (online), International Journal of Nonlinear Science, Vol. 8(2009) No. 3, pp. 274 278, (Received 6 November 2008, accepted 15 July 2009).

[2] B. Al Hamzah, N. Yebari (Existence and uniqueness of weak solution for weighted p- Laplacian Steklov problem). International Journal of Innovation and Applied Studies, Vol. 11 No. 1 Apr. 2015, pp. 69-76.

[3] A.R. El Amrouss, S. El Habib, N. Tsouli, Existence of solutions for an eigenvalue problem with weight. Electronic Journal of Differential Equations, Vol. 2010(2010), No. 45, pp. 110. ISSN: 1072-6691.

[4] S. A. Khafagy, Existence and uniqueness of weak solution for weighted p-Laplacian Dirichlet problem, Vol. 3, 2011, Online ISSN: 1943-023X, Jouranal of Advanced Research in Dynamical and Control Systems.

[5] S. A. Khafagy, Existence and Uniqueness of Weak Solution for Quasilinear Elliptic ( $p, q)$-Laplacian System. Global Journal of Pure and Applied Mathematics. ISSN 0973-1768 Volume 8, Number 4 (2012), pp. 465475.

[6] A. El Khalil, S. Kellati A. Touzani, On the spectrum of the p-biharmonic operator. 2002-Fez conference on Partial Dikerential Equations, Electronic Journal of Dikeren- tial Equations, Conference 09, 2002, pp 161170.

[7] El. M. Hssini, M. Massar, M. Talbi and N. Tsouli, Existence of solutions for a fourth order problem at resonance. Bol. Soc. Paran. Mat. (3s.) v. 322 (2014): 133142.SPM ISSN-21751188 , ISSN-00378712 in press.

[8] P. Lindqvist, Notes on the p-Laplace equation, NO-7491 Trondheim, Norway.

[9] P. Sorin Ilia s, P-laplacien à Poids Indéfini, Vol. LVIII, No. $2 / 2006$.

[10] E. El-zahrani , H. Serag, Existence of Weak Solutions for Nonlinear Elliptic Systems on $\mathfrak{R}^{\mathrm{N}}$, Electron. J. Diff. Eqns., Vol. 2006, No. 69, 1-10, 2006. 